Ciencia y Educación, Vol. 5, No. 3, septiembre-diciembre, 2021

ISSN (impreso): 2613-8794 • ISSN (en línea): 2613-8808

DOI: https://doi.org/10.22206/cyed.2021.v5i3.pp129-144

\title{
Recursos educativos en tiempos de COVID-19: la experiencia del Museo Interactivo Maloka
}

\author{
Educational resources amid the COVID-19 pandemic: \\ The Maloka Interactive Museum experience
}

Manuel Franco-Avellaneda a ORCID: 0000-0002-0895-8219

Lina Quijano ${ }^{\mathrm{b}}$ ORCID: 0000-0002-4497-8655

Angie Ariza-Porras ${ }^{c}$ ORCID: 0000-0002-8381-5146

Fabio Colmenares ${ }^{\mathrm{d}}$ ORCID: 0000-0002-2886-5407

Recibido: 25/04/2021・ Aprobado: 27/04/2021

Cómo citar: Franco-Avellaneda, M., Quijano Godoy, L., Ariza Porras, A. P., \& Colmenares, F. (2021). Recursos educativos en tiempos de COVID-19: la experiencia del Museo Interactivo Maloka. Ciencia y Educación, 5(3), 129-144. Doi: https://doi. org/10.22206/cyed.2021.v5i3.pp129-144

\section{Resumen}

El presente artículo recoge las experiencias de Maloka, un museo interactivo de ciencia y tecnología en Bogotá-Colombia que en tiempos de crisis tuvo que repensar sus estrategias de trabajo con la escuela, más allá de la presencialidad y con esto promover la apropiación social del conocimiento científico y tecnológico (ASC). En este contexto surge Aprende en Casa con Maloka: una alianza entre el museo y la Secretaría de Educación de Bogotá, que busca que los niños y las niñas de educación inicial y básica primaria, especialmente de contextos rurales y/o con vulneraciones socioeconómicas, puedan convertir sus hogares en ambientes de aprendizaje intencionados para el desarrollo de habilidades y actitudes de pensamiento científico.

Durante el programa, en el que se produjeron alrededor de 140 recursos educativos en diversos formatos, se desarrolló una evaluación formativa para identificar sus principales aprendizajes. En este proceso sobresalieron: (I) los retos de creación de recursos educativos en entornos en

\begin{abstract} and with socioeconomic vulnerabilities.
\end{abstract}

This paper presents the experience of Maloka, an interactive museum in Bogotá-Colombia, which in times of crisis had to redefine its strategies to relate with schools beyond faceto-face attendance in order to achieve processes of social appropriation of scientific and technological knowledge. In this context emerges the program "Aprende en casa con Maloka", an alliance between the museum and the Secretary of Education in Bogotá. This alliance seeks that children in initial and primary education can turn their homes into learning environments for the development of scientific thinking and skills, especially those from rural contexts

During the development and implementation of the program, the museum did a formative evaluation that sought to identify its main learnings. In this evaluative exercise, three reflections stood out: (1) the challenges of creating pedagogical resources in a context of digital gaps; (2) the role of interactive museums and their relationship

\footnotetext{
a Grupo de investigación de Apropiación Social de la Ciencia, Colombia. Correo-e: mfrancoavellaneda@gmail.com

b Grupo de investigación de Apropiación Social de la Ciencia, Colombia. Correo-e: lquijano@maloka.org

c Grupo de investigación de Apropiación Social de la Ciencia, Colombia. Correo-e: angiep.arizap@gmail.com

d Universidad del Rosario, Colombia, Correo-e: fabioandres2290@gmail.com
} 
donde persiste la brecha digital; (II) el rol de los museos en la configuración de ecosistemas de aprendizaje remotos que promuevan la ASC y (III) el fortalecimiento y la resignificación de las dinámicas entre la comunidad educativa y el museo, y en ellas la importancia de la familia.

Palabras clave: museo científico, educación, desigualdad social, ciencia, recursos educativos, pandemia.

\section{Introducción}

El cierre de las escuelas a nivel mundial debido a la pandemia por COVID-19 trajo consigo nuevos desafíos en términos pedagógicos, lo que abrió el camino a discusiones alrededor de la necesidad de promover transformaciones educativas. El confinamiento y la imposibilidad de adelantar procesos de enseñanza presenciales en las escuelas puso en marcha estrategias para una educación remota que permitiera a niños y niñas seguir el proceso educativo desde sus hogares. En ese sentido, gobiernos de todo el mundo pusieron a disposición una serie de recursos educativos, tales como programas de televisión y radio, plataformas digitales para la enseńanza, material educativo impreso, entre otros. De esa creciente oferta de recursos, el protagonismo lo tuvieron las herramientas digitales como principal estrategia para comunicarse e interactuar con los miles de estudiantes que estaban en sus hogares.

En este artículo expondremos la experiencia y los aprendizajes desde Aprende en Casa con Maloka ${ }^{a}$, un programa que surge de la alianza entre el museo interactivo y la Secretaría de Educación de Bogotá, cuyo propósito consistió en que los niños y las niñas de educación inicial y básica primaria de colegios distritales, especialmente de contextos rurales y/o con vulneraciones socioeconómicas, convirtieran sus hogares en ambientes de aprendizaje intencionados para el desarrollo de habilidades y actitudes de pensamiento científico. Con este fin, el artículo está dividido en los siguientes apartados: (I) una reflexión sobre el devenir de la educación en pandemia, tanto with the school in the configuration of learning ecosystems that promote the appropriation of science and technology; (3) the strengthening and new perspectives of the educational community dynamics in relation with the museum and the importance of families.

Keywords: Scientific Museum, education, social inequality, science, educational resources, pandemic.

en los establecimientos educativos como en los museos; (II) una descripción del programa Aprende en Casa; (III) una breve exposición de la metodología utilizada para la evaluación formativa; (IV) la presentación de resultados y $(\mathrm{V})$ unas conclusiones.

El acceso a clases virtuales y el uso de plataformas para la enseńanza requirió la tenencia de dispositivos electrónicos como tabletas, computadores o smartphones por parte de los docentes y sus estudiantes. El cambio de las relaciones pedagógicas que trajo la pandemia, mediadas principalmente por lo digital, también evidenció las desigualdades sociales, económicas y culturales, y el consecuente incremento de las brechas ya existentes en el aprendizaje e incluso el analfabetismo tecnológico en muchas regiones del mundo. En Latinoamérica y el Caribe el porcentaje de dispositivos disponibles con acceso a Internet no supera el 55\%, especialmente en zonas rurales y en regiones con altos índices de pobreza al interior de los diferentes países (García, 2020, p. 9).

A las barreras de acceso a la educación mediada por lo digital se suma el contexto de recesión económica que afronta la región, lo cual afecta directamente las condiciones sociales, nutricionales y psicológicas de niños, niñas, cuidadores y docentes. Adicionalmente, las dificultades de la mediación pedagógica que encuentran padres y cuidadores para acompańar el proceso educativo de niños y niñas han sido elementos que focalizan la atención, entre las cuales están: acercarse a las temáticas y las maneras como pueden ser enseñables, entender cómo aprenden mejor sus hijos y cómo acompañar ese proceso mientras los profesores están al

a. Más información en https://encasaconmaloka.org/ 
otro lado de las pantallas. De acuerdo con el informe de la García (2020), las estrategias de aprendizaje deben concordar con el ciclo escolar, por lo que se requiere especialmente en los más pequeños orientaciones concretas para el acompañamiento. Esto teniendo en cuenta que en la región el porcentaje de apoyo a actividades escolares disminuye en hogares pobres y en zonas rurales, debido a que los padres deben salir a trabajar, por un lado, en labores informales, y por otro lado, en las actividades propias del campo.

En el caso colombiano, implementar modalidades de educación a distancia es un desafío por las dificultades de acceso a Internet, especialmente en zonas rurales. Solo en Bogotá, una de las ciudades con menos brechas digitales, pero con un territorio rural mayor al 70\% (Secretaría Distrital de Planeación, 2019) se muestra un desbalance en las condiciones de vida y en las posibilidades de aprendizaje de niños y niñas en el campo, frente a sus pares de la ciudad. En efecto, como lo identificó el trabajo realizado por varias universidades de Colombia (Uniandes, 2020), los indicadores básicos de TIC en hogares en la Encuesta de Calidad de Vida en 2018 revelaron que el 50,8\% de los hogares en las ciudades tiene un computador (de escritorio, portátil o tableta), mientras en las áreas rurales solo el 9,6\% cuenta con estas herramientas tecnológicas.

Ahora bien, con relación a los teléfonos celulares inteligentes (smartphone) la situación es diferente, pues el 78,1\% de los colombianos tiene este tipo de teléfonos, aunque solo el 29,2\% utiliza Internet móvil. En cuanto a la conexión a internet en los hogares, uno de cada dos hogares colombianos que no cuenta con Internet afirma que la principal razón por la que no accede a este servicio es porque le resulta muy costoso (Ministerio de Tecnologías de la Información y las Comunicaciones, 2020).

Con el panorama de contingencia hasta aquí abordado, asociado con desafíos de mediación que ahora tienen padres y cuidadores y las dificultades de accesos a tecnologías, parece evidente la necesidad de replantear los espacios de la escolarización, los contenidos curriculares, las estrategias de enseñanza y los recursos materiales para la educación que ayuden a cerrar las brechas de aprendizaje que ha amplificado la actual contingencia (Porras, 2021). En este sentido, Inés Dussel (2020) propone el concepto de «domestización» de la escuela para comprender la actual situación, con base en tres ejes: los tiempos y espacios de la escuela y el aula; los contenidos y el peso de las tecnologías en esta situación.

En el primer eje, Dussel (2020), reconoce que la escuela es tanto un espacio material como una estructura comunicativa y que en la contingencia generada por la pandemia en la organización escolar sucedió una desaparición física del aula y con esto la posibilidad de aprender en colectivo entre pares sin la vigilancia adulta. En este nuevo escenario existe una demanda mayor de tiempo para los adultos (maestros, familias y cuidadores), pues ahora parece que están obligados a responder a cualquier hora y lugar.

En el segundo eje, los contenidos de la escuela, Dussel (2020) zanja la controversia entre seguir enseñando los contenidos establecidos o ajustar los programas a las circunstancias actuales. Argumenta que uno de los aprendizajes de la actual contingencia fue comprender que una clase no se trata de repartir tareas y corregirlas, sino que su objetivo debería orientarse a la posibilidad de configurar proyectos de conocimiento entre todos, en grupo, y construir condiciones para que cada uno pueda ir apropiándose de lenguajes y formas de pensar que propone la educación básica.

En el tercer eje, el peso de las tecnologías, identifica que históricamente la escuela se ha soportado en múltiples tecnologías: tiza, pizarras, imprenta, radio, etc. Estas permiten o imposibilitan ciertas relaciones; luego lo que se pone de manifiesto en la pandemia, más allá de la discusión puramente artefactual, es cuáles son los mejores soportes para sostener el proceso educativo, para atender el bienestar de todos los estudiantes y afrontar la desigualdad de condiciones.

En esta circunstancia, entendiendo que los museos, específicamente aquellos interactivos de ciencia y tecnología, son infraestructuras culturales corresponsables de procesos educativos en clave de divulgación, popularización y apropiación social del conocimiento (Franco-Avellaneda \& Arboleda, 2014), surge la pregunta sobre el papel de los museos interactivos de ciencia y tecnología en esta nueva realidad, en especial 
porque el nacimiento y la consolidación de estos espacios en las últimas décadas del siglo XX, en Latinoamérica, siempre estuvo acompañado de una retórica de complemento y, en algunos casos, de suplencia de la escuela (Franco-Avellaneda, 2013b). Además de esa demanda, que hoy ya no es de posicionamiento sino de subsistencia, puede ser una oportunidad para entablar diálogos permanentes con la escuela, pues ambas instituciones viven una contingencia. En efecto, los museos, en específico los interactivos de ciencia, con la poca o nula presencia de visitantes en sus grandes edificaciones, experimentan una inviabilidad de su propuesta de ofrecer una experiencia directa con los fenómenos naturales, físicos y tecnológicos, a través de las exposiciones interactivas.

\section{Reorientación de los museos en medio de la pandemia}

Varias investigaciones han señalado que los museos interactivos existen porque existe la escuela (Franco-Avellaneda, 2013a; Pérez-Bustos, 2010). Buena parte de los recursos de los museos interactivos provienen de actores relacionados con el sistema educativo o interesados en él, pues el público escolar es el que más frecuenta estos escenarios, como lo muestra Pérez-Bustos (2009). Los escenarios para la popularización del conocimiento científico y tecnológico, museos interactivos de ciencia, aunque inicialmente toman distancia de la escolarización y buscan diferenciarse de la escuela, terminan por incorporarse en el sistema educativo. Esto sucede porque mientras se legitiman como escenarios ideales frente a la crisis de la educación (Coombs, 1971), y entran a competir por recursos públicos y privados destinados a la formación en ciencia y tecnología, también se convierten en una manera más de curricularizar la sociedad, al utilizar y reforzar las mismas lógicas del sistema educativo a las que se refiere Martínez-Boom (2004): la expansión horizontal (intentan llegar a mayor cantidad de públicos posibles) y vertical (cada vez es más usual que ofrezcan experiencias especializadas).
Si bien esa fue la dinámica que marcó el desarrollo y posicionamiento de la mayoría de museos interactivos en el contexto colombiano y, podríamos decir, latinoamericano (Franco-Avellaneda, 2013b), en la última década, y con especial rapidez en el marco de la pandemia, se fortaleció la complementariedad entre museo-escuela y, en general, hubo un mayor peso en el rol del museo como articulador entre ciencia y sociedad. En efecto, más que competir con la escuela como epicentro para el acceso al conocimiento y la cultura, estos espacios poco a poco se reafirman como una infraestructura cultural que enriquece los procesos de enseñanza y de aprendizaje de manera significativa, siempre que tengan como punto de partida el diálogo con las instituciones educativas sobre la base de la realidad escolar. Esto ha implicado reconstruir una relación de confianza entre personas (maestro y agente educativo del museo), entre instituciones (museo, secretarías de educación, instituciones educativas) y en los encuentros y experiencias desarrollados en sí mismos (asegurando que sean significativos).

Durante la crisis generada por el Covid-19, los museos cerraron sus puertas por varios meses del año 2020, lo que implicó pensar en posibilidades de acción a lo largo del confinamiento (Elizarrarás, 2020). De hecho, los museos interactivos y, en particular, el Museo Interactivo Maloka en Bogotá (Maloka), vivieron un replanteamiento de su rol social porque el boom de los science center, que les sirvió de inspiración, entró en crisis (Beetlestone, Johnson, Quin \& White, 1998; Bradburne, 1998; Franco-Avellaneda, 2013b); por un lado, por la inutilidad de los artefactos y las exposiciones mientras el museo estaba cerrado, pues la pandemia implicó un giro en el museo interactivo, desplazando a los artefactos de las salas de exposiciones por experiencias no presenciales difundidas en redes sociales o a través de la conceptualización, el diseño y la distribución de recursos educativos. Por otro lado, sus grandes construcciones con salas de cine de gran formato, en medio de las restricciones de movilidad de personas y de eventos masivos, se convirtieron en una carga difícil de mantener durante tanto tiempo de cierre, pues el equipo técnico que hacía funcionar esta infraestructura ya no se podía sostener. 
Así las cosas, resulta conveniente recoger las experiencias de un museo interactivo que, en tiempos de crisis, se reconfigura más allá de sus muros; en específico, se presenta el diseño y la puesta en marcha del programa Aprende en Casa con Maloka, el cual desarrolla y distribuye recursos educativos impresos para niños y niñas que en muchos casos no pueden acceder a herramientas y soportes digitales. Este programa parte de entender que un proceso educativo tiene como base el acompañamiento, no únicamente la enseñanza, así como de las posibilidades de mediación que tiene este tipo de recursos cuyo propósito es complementar el desarrollo escolar en tiempos de pandemia. Esto significa privilegiar acciones orientadas en las preguntas sobre qué necesitan niños y niñas y sus familias, y para qué lo necesitan.

\section{Aprende en Casa con Maloka}

El programa Aprende en Casa con Maloka nació como una alianza entre el Museo Interactivo Maloka y la Secretaría de Educación de Bogotá, buscando que niños y niñas de educación inicial y básica primaria, especialmente aquellos de contextos rurales y con vulneraciones socioeconómicas, convirtieran sus hogares en ambientes de aprendizaje intencionados para el desarrollo de habilidades y actitudes científicas. En ese sentido, los contenidos son una excusa para recolectar información, organizar datos, analizar evidencias, etc.

Se dirige este programa a niños y niñas de educación inicial y primaria, reconociendo que en la básica primaria sientan las bases para el desarrollo de su pensamiento, que se hace más complejo en etapas posteriores del proceso educativo y, por tanto, dichas bases deberían ser adquiridas con solidez para aumentar las posibilidades de acceder y culminar de forma exitosa la educación básica secundaria, media y superior. Priorizar la primaria en la educación científica es una manera de contribuir para cerrar la brecha que se abre y se profundiza en la estructura piramidal que adopta el acceso a la educación. Igualmente, la incidencia en el desarrollo del pensamiento científico en los primeros años de escolarización se hace central para la calidad de la educación (OECD, 2016).

A partir de esta intencionalidad educativa, la propuesta didáctica reconoce tres actores clave: niños y niñas, maestros y familias, para los cuales se desarrollan las acciones y los recursos, tanto digitales como impresos, descritos en la Tabla 1.

\section{Tabla 1}

Materiales e interacciones según tipos de actores. Programa Aprende en casa con Maloka, 2020

\begin{tabular}{|c|c|c|}
\hline Actores & Recursos & $\begin{array}{l}\text { Espacios de } \\
\text { interacción remota }\end{array}$ \\
\hline Niños y nińas & $\begin{array}{l}\text { Guías de trabajo } \\
\text { en casa } \\
\text { Podcast } \\
\text { Videos }\end{array}$ & \multirow{3}{*}{$\begin{array}{l}\text { - Call center para } \\
\text { preguntas } \\
\text { - Franjas online }\end{array}$} \\
\hline Maestros & $\begin{array}{l}\text { Guías digitales } \\
\text { para acompañar } \\
\text { los procesos de } \\
\text { los estudiantes }\end{array}$ & \\
\hline Familias & $\begin{array}{l}\text { Guías para } \\
\text { acompañar el } \\
\text { proceso de niños } \\
\text { y niñas }\end{array}$ & \\
\hline
\end{tabular}

Estas acciones configuran una presencialidad remota, pues contemplan, como parte fundamental de su implementación, la entrega de recursos editoriales en las casas de niños y niñas, al considerar las brechas digitales y además aprovechar las infraestructuras tecnológicas en el contexto, que principalmente usan el celular como medio de comunicación, para poner en acción el acompañamiento de los estudiantes durante el cierre de las escuelas y así apoyar el proceso educativo. La idea de presencialidad remota también refiere a los encuentros que se proponen, en tiempos y espacios determinados (programación de franjas online vía Facebook) o no (call center), para acompañar las preguntas y cuestiones de los diferentes actores involucrados en el proceso educativo. La clave de estos espacios y materiales en lógica de presencialidad remota consiste en que se permiten interacciones entre personas situadas en diferentes zonas de una ciudad 
como Bogotá, que cuenta con veinticinco colegios rurales ${ }^{b}$. También se logran integrar múltiples medios de comunicación e interacción pedagógica (video, podcast, Facebook live, call center, material editorial y recursos digitales). Además, facilita interacciones entre colegios, docentes, estudiantes y familias, cuestión que parece diluirse en el contexto de pandemia.

\section{Metodología de la evaluación formativa}

De manera transversal al diseño e implementación de este programa, se realizó un ejercicio de evaluación formativa con el objetivo general de identificar las fortalezas y oportunidades de mejora del diseño e implementación del programa desde la perspectiva de sus participantes, con el fin de retroalimentar constantemente sus acciones para tomar decisiones pertinentes durante su realización. Siguiendo a Tejedor (2000), la evaluación formativa "permite establecer una interacción continua entre la evaluación y la acción educativa" (p. 325) y genera orientaciones pertinentes dirigidas a la optimización del programa.

La evaluación planteada fue principalmente de carácter cualitativo, pues este tipo de investigación nos permite dar cuenta de este fenómeno, de manera que pueda ser leído "desde la complejidad que supone un proceso social contextualizado, denso y multidimensional como la Apropiación Social de la Ciencia y la Tecnología" (Daza-Caicedo et al., 2017). Es así como uno de los retos de la evaluación fue el diseño de instrumentos cualitativos de evaluación que pudieran ser aplicados de manera remota y digital, que despertaran el interés por participar de los públicos y que dieran cuenta de la multiplicidad de recursos educativos y de actores que se apropian de ellos.

Desde una perspectiva metodológica, la evaluación se enmarcó en los postulados de la evaluación de la implementación, desde la cual se analiza el funcionamiento del programa, verificando si durante su desarrollo se cumplen los objetivos y el diseño previsto, además de identificar desde la perspectiva de los principales actores involucrados los principales logros y las dificultades en los diferentes aspectos que constituyen el programa. Debido a la cantidad de familias, estudiantes y docentes que hicieron parte de la población focalizada para este programa, se realizó un muestreo no probabilístico tipo intencional, desde el cual se seleccionaron a los participantes de las diferentes técnicas de evaluación propuestas.

De esta forma, se enfocó en tres elementos clave del programa: la línea editorial (guías y anexos), la línea virtual (videos, podcasts y franjas online) y la estrategia de acompañamiento pedagógico (micrositio y call center). Las acciones ejecutadas para tal fin se realizaron en diferentes momentos de la implementación del programa para contar con insumos durante la creación de los recursos, así como para ir obteniendo las perspectivas frente al uso y la apropiación por parte de estudiantes, docentes y familias.

Durante el desarrollo del programa (de mayo a noviembre de 2020) se crearon espacios de discusión con docentes, estudiantes y familias de tal forma que se pudiesen recoger las percepciones sobre todos los recursos educativos del programa y la estrategia de acompañamiento pedagógico, así como también reflexiones sobre otros temas, tales como el componente logístico del programa. De este modo, se desarrollaron una serie de estrategias para la recolección de información, esto teniendo en cuenta las dificultades metodológicas de la implementación de algunos instrumentos de forma no presencial.

- Grupos focales: realizados virtualmente durante diferentes momentos del año, estos grupos focales se realizaron con familias y estudiantes de las instituciones educativas focalizadas por el programa, las cuales fueron seleccionadas de forma intencional, siendo familias que pudiesen conectarse virtualmente y que tuviesen disposición de tiempos. Estos grupos focales buscaron identificar oportunidades de mejora del material entregado, posibilidades de uso que se le dio a este material y la aceptabilidad hacia el programa.

b. Aprende en Casa con Maloka llega al 70\% del total de matrícula rural de Bogotá. 
- Encuesta a docentes: realizada a través de un formulario digital durante los dos espacios de encuentro con los y las docentes participantes realizados durante la implementación del programa; con estas encuestas se buscó identificar el interés hacia el programa y hacia los recursos entregados, así como la pertinencia del material entregado y recoger la retroalimentación sobre el programa y los diferentes recursos diseñados.

- Entrevistas a rectores: este instrumento se realizó de forma telefónica a 33 rectores de 43 instituciones educativas, al finalizar el proyecto, que buscó identificar la perspectiva de los rectores frente a la ejecución del programa y el material educativo entregado a los estudiantes, así como identificar oportunidades de mejora.

Esta diversidad de información recolectada se clasificó en categorías de análisis para identificar las principales oportunidades de mejora del programa y poder hacer adaptaciones o ajustes a la propuesta de forma pertinente. Finalmente, la información recolectada durante la implementación de todo el programa, así como la sistematización de los ajustes propuestos, fueron los principales insumos bajo los cuales se perfilaron los principales resultados de esta investigación.

\section{Resultados de la evaluación formativa del programa}

\section{El museo de ciencias como escenario para la educa- ción en tiempos de confinamiento}

Como resultado del confinamiento, Maloka, así como otros espacios educativos y culturales, ha venido proponiendo recursos educativos para niños y niñas que no solo promueven el aprendizaje y el interés por la ciencia y la tecnología, sino que permiten consolidar alianzas entre el museo y la escuela. De esta manera, este centro de ciencias ha consolidado recursos educativos desde la presencialidad remota, a través de diversas estrategias que van desde lo digital hasta lo material, aportando a las necesidades de las escuelas de llevar materiales educativos a sus estudiantes sin acceso a Internet.
Para cumplir con los objetivos del programa se asumen una serie de retos según las características geográficas y sociodemográficas de la población focalizada, esto sin contar los retos que Maloka, como museo de ciencias, debe enfrentar para la ejecución de un programa bajo las circunstancias de aislamiento. Definir la población objetivo del programa representó un reto, ya que, si bien existe una focalización en las instituciones educativas rurales de Bogotá o con condiciones de vulneración socioeconómica para definir las familias que recibieron los recursos editoriales en sus casas, también existe un público ampliado que accedió a los recursos de manera digital.

Otro reto, que procede del anterior, está relacionado precisamente con la realidad de la brecha digital, la oportunidad de acceso a materiales virtuales o digitales y la necesidad de establecer acciones y estrategias enfocadas en que se construya una presencialidad remota incluyente y no solo dependiente de lo digital. Resolver esta serie de retos, y los demás asociados a la conceptualización, el diseńo y la producción de los materiales y recursos pedagógicos y su apropiada distribución, entre otras acciones, requiere pensar en el papel que juega la producción de materiales digitales en un mundo de acceso limitado a canales virtuales o digitales de acceso a la información. Consecuentemente, una de las primeras exploraciones que se debía realizar está relacionada con una caracterización, especialmente en términos de acceso a tecnología o conectividad, para entender mejor el escenario retador y definir las estrategias del programa.

El escenario de implementación de Aprende en Casa con Maloka refleja, sin duda, un problema de acceso a lo digital. Esto se confirma con la encuesta telefónica realizada a 14,000 familias de las 26,000 registradas en el programa, de las cuales el $44 \%$ reporta no tener acceso a Internet, frente a un 55\% que menciona tener acceso. Sin embargo, a pesar de tener acceso a Internet, más de la mitad de estas familias $(4,503)$ no posee herramientas informáticas para acceder al servicio. 


\section{Tabla 2}

Resultados de la encuesta telefónica a las familias del programa Aprende en Casa con Maloka, 2020

\begin{tabular}{cccc}
\hline & $\begin{array}{c}\text { Sin acceso a } \\
\text { Internet }\end{array}$ & $\begin{array}{c}\text { Con acceso a } \\
\text { Internet }\end{array}$ & \\
\hline $\begin{array}{c}\text { Sin acceso a } \\
\text { herramientas } \\
\text { informáticas }\end{array}$ & 5727 & 4504 & $\mathbf{1 0 2 3 1}$ \\
\hline $\begin{array}{c}\text { Con acceso a } \\
\text { herramientas } \\
\text { informáticas }\end{array}$ & 668 & 3619 & $\mathbf{4 2 8 7}$ \\
\hline & $\mathbf{6 3 9 5}$ & $\mathbf{8 1 2 3}$ & \\
\hline
\end{tabular}

De tal forma, las diversas estrategias planteadas desde el programa se cruzan con las singularidades de las realidades sociales y económicas de la comunidad educativa. Las dificultades de acceso a medios digitales de una gran parte de ella (véase la Tabla 2) han hecho que el material impreso sea el único tipo de recurso al que pueden acceder. Por otro lado, la intermitencia del acceso a Internet o a las herramientas informáticas provoca que el uso de contenido complementario a las guías impresas, como los podcasts, videoclips o los encuentros online no sea constante ni sincrónico. Dentro del programa ha sido fundamental profundizar en esta relación entre el diseño de materiales pedagógicos que tendrán sus respectivas versiones digitales y físicas. Así, es posible hablar no solo del diseño y de la distribución de materiales desde lo digital, sino de la integración de diferentes realidades en una presencialidad remota.

Aprende en Casa, diversas estrategias ante la presencialidad remota: distintos canales según el público

En este contexto, Aprende en Casa con Maloka es un programa que ha desarrollado diferentes recursos para el fortalecimiento de habilidades y actitudes científicas. Estos se han creado a partir de una pregunta o de un tema de interés desde donde el estudiante configura sus aprendizajes, los pone en práctica por medio de ejercicios de experimentación o exploración y promueve estrategias de comunicación de sus experiencias a otras personas.

Para la mayoría de los rectores consultados, el contenido de las cartillas entregadas a las familias focalizadas es pertinente por tres razones: en primer lugar, la entrega de recursos editoriales impresos a las familias está en concordancia con las dinámicas que ha traído la actual pandemia y, articulado con esto, el material abrió la posibilidad de que los hogares de los niños y las niñas sean estaciones de exploración, lo cual propicia que las casas adquieran el estatus de ambientes de aprendizaje de ciencia y tecnología. Ante esto, uno de los rectores menciona:

En general el apoyo es importante. Cualquier acompańamiento que garantice que se continúen supliendo las falencias del nivel central para garantizar gestión, materiales educativos o dispositivos tecnológicos, son apoyos que son bien recibidos. Hay muchos problemas de conectividad, el apoyo en físico permite mejorar las condiciones de las estrategias con los estudiantes. (Rector entrevistado)

En segundo lugar, el diseño de las cartillas se adapta a las edades e intereses de los niños y las niñas. Muchos de los rectores aluden a que el material es de fácil comprensión para niños, niñas y cuidadores, y esto posibilita el trabajo en familia. Y, en tercer lugar, el material permitió la articulación con el trabajo de aula de algunos docentes, siendo un complemento no solo para el desarrollo de habilidades y actitudes científicas, sino también para el fortalecimiento de algunas habilidades blandas y otros procesos de producción y comprensión en tanto se promueven la lectura y la escritura.

Es así que, a partir de los intereses de los niños y las nińas, aumenta el interés por utilizar los recursos educativos propuestos, tal como lo menciona uno de los niños participantes en el programa:

Me gustó las actividades, los experimentos, todo porque es una cosa diferente que vamos a aprender [...]. No se trata de hablar de los romanos, de matemáticas, de nada de eso, sino que es como unas 
actividades que nos ocupa a nosotros el tiempo y hay cosas bonitas para hacer. (Grupo focal niños. Octubre de 2020)

Sin trazar una separación radical entre los recursos escolares y los que se ofrecen desde el programa, se abre la pregunta sobre los tipos de uso y aprovechamiento diferencial en el hogar de los recursos de Aprende en Casa. Desde la perspectiva de los niños y las niñas participantes del programa, especialmente de quienes han recibido los recursos editoriales impresos y participaron en los grupos focales, los materiales cumplen una doble función: por un lado, sirven para el aprovechamiento del tiempo libre pero, por el otro, el uso de estas guías está ayudando a fomentar la curiosidad, así como el trabajo colaborativo entre los estudiantes y sus familias.

En general, familias y estudiantes comparten percepciones muy positivas de los recursos educativos. El elemento que más destacan es la experimentación y el juego como parte de los recursos educativos. En los grupos focales, las familias definen los recursos como didácticos, conscientes, divertidos, creativos y llamativos. El diseño genera mucha atracción y el uso de los colores, las imágenes y las figuras ayuda a captar la atención de los niños y las niñas. Por último, cabe resaltar que los niños y las niñas disfrutan de la narrativa de las guías y sienten que es mucho mejor utilizar las guías siguiendo a los personajes que articularon la narrativa del programa: Lúa, Tomás y Greta.

En cuanto a los docentes, uno de los resultados más importantes está relacionado con el alto componente experimental que se presenta en las guías para estudiantes. Los docentes que han participado en los encuentros mencionan que el hecho de que las guías se concentren en actividades y experimentos puede ayudar tanto a la motivación de los estudiantes, como a la posibilidad de pensar en articulaciones con el currículo. Adicionalmente, para los docentes los recursos tienen posibilidades de implementación con los estudiantes debido a los temas atractivos que tratan, así como a la forma en la que se presentan a los niños y las niñas en cuanto al uso del lenguaje. Sin embargo, en un comienzo, para ellos la articulación con el currículo no es clara, lo que puede dificultar la vinculación directa con los planes de aula.

Este último punto resulta importante para el equipo pedagógico del programa. Si bien en las guías para docentes se incluye una matriz detallada de sugerencias para desarrollar planes de aula a partir de los recursos pedagógicos, los docentes sienten que esto puede no ser suficiente en un doble sentido: (1) para propiciar la articulación con el currículo se requiere conocer a detalle el material impreso que llega a los niños y las niñas; esto implica que todos los docentes reciban los recursos impresos previamente (2) aunque se presenta en la propuesta de plan de aula de las guías para docentes, su apropiación depende del tipo de habilidad y actitud por desarrollar en cada guía y del área temática o de la disciplina desde la que se quiera abordar. En otras palabras, aunque el material pedagógico ha sido diseñado con la intención de brindar la mayor cantidad posible de posibilidades y autonomía de articulación curricular a los docentes, aún se debe fortalecer con ellos estos vínculos.

$\mathrm{Al}$ respecto, desde la perspectiva de los rectores, el material se presta para articularse con las actividades curriculares de los docentes, especialmente como un apoyo pedagógico. Varios de los rectores consultados han visto que los docentes de sus colegios están utilizando el recurso educativo en sus clases, por lo cual ven que hay un potencial educativo complementario en el recurso educativo. Sin embargo, otro de los aspectos a mejorar que más resaltan los rectores es la necesidad de que Maloka pueda trabajar directamente con los docentes de las IED brindando asistencia o capacitación para el uso de los recursos educativos, hecho que se pretende mejorar con la implementación de una estrategia de acompañamiento y capacitación docente en la segunda fase del programa. Si bien existen algunos rectores que reconocen que el material en sí mismo brinda herramientas para que los docentes adelanten una articulación curricular suficiente, otros rectores mencionan que se deben definir espacios específicos para que Maloka apoye ese proceso de articulación curricular a través del trabajo directamente con los docentes. Los rectores recomiendan que los 
docentes puedan no solo consultar los recursos a través de la página web, sino tenerlos en físico y contar con espacios para familiarizarse con ellos y de esta manera preparar y planear mejor sus actividades.

Los docentes, adicionalmente, aprovechan los espacios de encuentro con el museo para sugerir temáticas a través de las cuales se pueden fortalecer habilidades y actitudes científicas. En un ejercicio de propuesta y priorización de temas a través de un formulario de satisfacción de Google, los docentes jerarquizaron los temas, como se aprecia en la Tabla 3.

\section{Tabla 3}

Priorización de temáticas para los materiales por parte de los docentes, 2020

\begin{tabular}{cc}
\hline Temáticas-áreas propuestas & Cantidad \\
\hline Experimentación en general & 20 \\
\hline Ciencias y tecnología & 16 \\
\hline Cuerpo humano & 7 \\
\hline Empatía-Convivencia-Sociales & 7 \\
\hline Matemáticas & 6 \\
\hline Medio ambiente & 6 \\
\hline Interdisciplinar & 5 \\
\hline Arte, color y formas & 3 \\
\hline Sonidos & 3 \\
\hline Tiempo y espacio-astronomía & 3 \\
\hline Creación narrativa - lenguaje & 3 \\
\hline Otros idiomas (inglés) & 2 \\
\hline Formación docente & 2 \\
\hline Para padres (talleres) & 1 \\
\hline Diversidad - material para niños y & 1 \\
\hline nińas con discapacidades & \\
\hline
\end{tabular}

Ligado a lo anterior, es necesario reflexionar acerca de que la presencialidad remota, además de poner en evidencia ciertas complejidades de los contextos y las realidades que viven las familias e instituciones priorizadas, devela las necesidades de los docentes para fortalecer sus estrategias y esfuerzos educativos bajo la situación de aislamiento y confinamiento. Por ejemplo, si se piensa que la presencialidad remota implica el uso de recursos y materiales pedagógicos digitales y análogos, esto exige también una armonización, tanto para el diseño y la distribución (aseguramiento al acceso) como para la articulación pedagógica y curricular entre cuatro actores: los estudiantes, la familia, los docentes y el museo.

\section{El protagonismo de las familias en la escuela}

En tiempos de confinamiento, la familia ha jugado un rol importante en el acompañamiento de las actividades propuestas en el programa. Es así como los padres o cuidadores se han constituido en asistentes en los ejercicios de experimentación y exploración, lo cual lleva al afianzamiento de los lazos y promueve un diálogo de saberes entre niños, niñas y adultos, tal como lo afirman los estudiantes:

Primero hicimos el gel [antiséptico], con ayuda de mi mamá lo hicimos. Luego hicimos la estación de desinfección, para cuando mi mamá o mi papá llegaran del trabajo. [...] A veces nos parecía chistoso porque mi mamá se equivocaba en algunas partes. También mi hermana, ella tiene 9 años [...]. Ella me ayudaba decorando unos cartelitos. (Grupo focal con niños, 2020)

Este es sin duda uno de los resultados más importantes por resaltar: los recursos diseñados desde el programa han brindado la oportunidad de articular los roles de estudiantes y familias para el desarrollo de las guías. En la totalidad de los casos que han participado en nuestros espacios de discusión, como los grupos focales, los niños y las niñas han afirmado que han recibido apoyo o compañía de sus padres, madres o cuidadores en el proceso de realización de las actividades o los experimentos. Aunque seguramente hay casos en donde esta articulación estudiante-familia no se presente, resulta positivo encontrar que las guías efectivamente estimulan este tipo de interacciones y usos en el contexto familiar. A partir de allí, se puede reflexionar que este tipo de articulación (así como la 
articulación estudiante-docente y familia-docente) son pilares que se deben seguir trabajando a la hora del diseño de este tipo de recursos que fomentan el fortalecimiento de habilidades y actitudes desde la presencialidad remota.

Cabe resaltar que los estudiantes también se sienten motivados e interesados en desarrollar las actividades de forma autónoma, como lo indicaron en los grupos focales. Esto significa que, así no estén acompañados por un adulto, se sienten atraídos por las guías para desarrollar las actividades, lo que viene a mostrar que es posible desarrollar procesos de aprendizaje autónomo por medio de las guías. Un elemento de especial atención es que los estudiantes sienten que estos recursos educativos les han permitido relacionarse mejor con sus familias, ya que la mayoría de las veces realizan las actividades con ellas. Para ellos el principal acompañante son sus padres, madres o cuidadores, con quienes revisan las guías, interpretan las instrucciones y ejecutan los experimentos. Desde esta perspectiva, los niños y las niñas sienten que los recursos educativos les dan la opción de desarrollar las actividades acompañados o por su cuenta.

Por lo anterior, en estos programas y proyectos de presencialidad remota debemos entender a la familia como un agente central en los procesos educativos. Bajo este escenario, no se puede asumir que las familias cumplirán un rol de supervisores, o en casos más preocupantes, como figuras ausentes, sino que son un agente en sí mismo que puede dar acompañamiento y robustecer el proceso de fortalecimiento de las habilidades y actitudes científicas. Del rol activo y central que asume la familia en el desarrollo de esta presencialidad virtual en este programa hemos encontrado que es necesario fortalecer la relación familia-docentes. En el marco del programa, parece que esta relación se diera exclusivamente en términos de consultar temas logísticos, como si las familias y los estudiantes recibieron el material o si las actividades serán evaluadas; pero aún se debe fortalecer la conexión entre la familia y los docentes como promotores armónicos y corresponsables del desarrollo y fortalecimiento de habilidades y actitudes científicas de niños y niñas.

\section{El desarrollo de habilidades y actitudes científicas en niños y nińas en escenarios ampliados}

Aprende en Casa con Maloka no es solo una apuesta ante las dinámicas de enseñanza de las ciencias, sino que busca que el pensamiento cientííico sea una forma de posicionarse ante el mundo para comprender la realidad. Esta apuesta nace teniendo en cuenta las particularidades del museo como espacio de educación no formal y el diálogo que teje con la escuela. De acuerdo con Harmen (2008), citada por Furman (2016), el desarrollo de un pensamiento científico implica tener la capacidad de desarrollar curiosidad, satisfacerse de encontrar respuestas a los propios interrogantes, flexibilizar el pensamiento siendo capaz de escuchar a los otros y tener el deseo de seguir aprendiendo. Este pensamiento científico tiene que ver con lo emocional, pero también está permeado por los intereses, las actitudes, las creencias e imaginarios de las personas.

Los niños y las niñas presentan, desde pequeños, capacidades asociadas al pensamiento científico, pero necesitan ser desarrolladas y potenciadas a través de la educación con otros. La formación de pensamiento científico en la escuela es fundamental, dado que promueve la curiosidad, incentiva el interés sobre el entorno, media en los procesos de exploración del mundo y permite que los niños sean conscientes de los procesos que emprenden y las transformaciones que generan sobre su comprensión de la realidad (Furman, 2016). Al respecto, los docentes destacan que las guías y sus actividades permiten hacer profundizaciones en diferentes temáticas, de tal suerte que el desarrollo de las actividades ya tiene unas habilidades y actitudes específicas que busca fortalecer, por lo que se presta para hacer una articulación curricular más fácilmente.

De esta forma, desde el programa se comprenden las habilidades científicas como la facultad de una persona de aplicar procedimientos cognitivos en tareas concretas, los cuales están relacionados con las formas en cómo se construye pensamiento científico. De esta manera, las habilidades posibilitan a las personas 
aplicar procedimientos para la solución de problemas de la vida cotidiana, siendo una herramienta que permite el desarrollo cognitivo, emocional y social desde las primeras etapas de la vida.

Por su parte, la actitud es una disposición ante el mundo, siendo constructos que median nuestras acciones; al hablar de actitudes científicas nos referimos a la disposición hacia las personas, situaciones y prácticas implicadas en el aprendizaje de la ciencia y que permiten configurar un pensamiento científico.

Teniendo en cuenta la importancia de las habilidades y actitudes científicas y el alto impacto que tiene la ciencia y la tecnología en la forma como vivimos, es indispensable que los museos junto con la escuela sean agentes formadores de pensamiento científico en los niños y las niñas. Esto implica entablar diálogos entre la escuela y el museo que permitan desarrollar escenarios más amplios para la enseńanza y el aprendizaje de las ciencias, a fin de promover en los estudiantes un abanico de actitudes y habilidades frente a la ciencia y la tecnología que permitan el aprendizaje a lo largo de la vida. Es así como los recursos educativos desarrollados por Maloka en el marco del programa en mención contemplan la configuración de la casa como laboratorio de exploración en el que la ideación y puesta en marcha de actividades alrededor del juego, la exploración y la experimentación promoverán las actitudes y habilidades científicas en los niños y las niñas.

\section{Perspectiva institucional: seguimiento con rectores y líderes de los colegios (Instituciones de Educación Distrital - IED).}

\section{- Percepciones generales del programa por parte de directivos docentes}

Las impresiones y percepciones que los rectores tienen sobre el programa Aprende en Casa con Maloka y que expresaron en las entrevistas son positivas; en su mayoría, para referirse a la estrategia se alude a calificativos como interesante, pertinente o excelente. De igual forma, se mencionan las posibilidades de uso que ha tenido el programa en épocas de confinamiento y la pertinencia de situar la apuesta en los hogares de los niños.
Las percepciones no solo se limitan a la apuesta pedagógica del programa, sino también a otros aspectos que lo componen, como el proceso de distribución del material o la articulación con familias y docentes.

\section{- Programa vs. contexto}

Uno de los elementos que más mencionaron los rectores, que impacta en la implementación del programa, es el contexto de las familias a las cuales va dirigido. La falta de conectividad o las dificultades en el acceso a herramientas tecnológicas es uno de los elementos que influye en el desarrollo de este tipo de programas. A pesar de las limitaciones para la enseñanza a distancia, este programa divisa otras posibilidades de aprendizaje no presencial. Ante esto, uno de los rectores menciona:

En general el apoyo es importante. Cualquier acompańamiento que garantice que se continúen supliendo las falencias del nivel central para garantizar gestión, materiales educativos o dispositivos tecnológicos, son apoyos que son bien recibidos. Hay muchos problemas de conectividad, el apoyo en físico permite mejorar las condiciones de las estrategias con los estudiantes. (Rector entrevistado)

\section{- Aceptabilidad}

Los rectores consideran que el material es apropiado, adecuado y de calidad. El diseño de las cartillas es visualmente atractivo tanto para los estudiantes como para docentes y cuidadores, siendo un elemento que genera motivación para ser usado.

En cuanto al contenido, la posibilidad de abordar el pensamiento científico desde las habilidades y las actitudes permite un trabajo interdisciplinario y articulado a proyectos de aula o proyectos transversales que se vienen adelantando en las instituciones. De esta forma, los recursos educativos del programa (guías, podcast o videos) han sido usados de diferentes formas por los docentes. Ante esto, una docente de educación inicial menciona "nosotros veníamos desarrollando el proyecto Exploradores en 
casa y llega Maloka a complementar estas actividades que veníamos trabajando en el colegio" (Grupo focal docentes, 2020). Asimismo, otra maestra de básica primaria menciona las articulaciones que ha encontrado entre su planeación de aula y el material de Aprende en Casa, "las cartillas de Maloka fortalecen los contenidos que abordamos con los niños ya que los contenidos van acordes a los que se aborda en el ciclo" (Grupo focal docentes, 2020).

\section{- Articulación entre Maloka y las Instituciones Educativas}

Los rectores consideran que se puede profundizar la articulación entre Maloka y las Instituciones Educativas para fortalecer elementos logísticos y de acompañamiento a familias y a docentes para el uso de los recursos educativos. A continuación, se exploran las percepciones de los rectores frente a la relación entre Maloka y las IED focalizadas, de cara al desarrollo del programa.

\section{- Articulación para aspectos logísticos}

Los rectores encontraron, en su mayoría, que el programa, a pesar de ser una iniciativa significativa y pertinente, tiene varios retos a nivel logístico. Es importante resaltar que los rectores consideran que gran parte de los inconvenientes logísticos que se pueden presentar, como que no todos los estudiantes reciban los recursos físicos a tiempo, pueden evitarse si se da una mayor articulación entre Maloka y los colegios. Los rectores aseguran que la logística de entrega de los recursos educativos a las familias funcionaría mejor si las entregas las hacen directamente los colegios.

Esta articulación logística requeriría de un acompańamiento por parte de Maloka con profesionales o asistentes que tengan claridad sobre el manejo de los listados para que apoyen el proceso de entrega en las instituciones educativas. Esto implica que Maloka apoye con profesionales, con manejo de listas, con articulación para fechas de entrega y con apoyo para las entregas.

\section{- Acompañamiento a las familias y seguimiento}

Los rectores afirman que en ocasiones es difícil lograr articular a las familias y los cuidadores en estos procesos debido a varios factores. El primero tiene que ver con las particularidades del contexto. Por ejemplo, si las familias se encuentran en contextos rurales, en ocasiones las familias pueden tener inconvenientes para recoger los recursos educativos. Otro ejemplo está relacionado con familias que, por su ocupación o por cuestiones de distancia o de cambio de domicilio, tienen inconvenientes para cumplir con las fechas pactadas para las entregas. Los rectores consideran que un acompañamiento por parte de Maloka podría ayudar a solventar estos inconvenientes. Se recomienda que las familias reciban capacitaciones o acompañamiento directo para el uso de los recursos con sus hijos.

\section{Discusión y conclusiones}

Las restricciones de movilidad a raíz de la actual pandemia han ofrecido la posibilidad de volver a pensar en lo que Falk \& Dierking (2018) denominaban ecosistemas de aprendizaje. La concepción del aprendizaje como un proceso de configuración de saberes, habilidades, percepciones y actitudes que sucede a lo largo de la vida y que involucra diversos actores en espacios formales, no formales e informales de educación, exige esfuerzos constantes de lectura del entorno, del diseño de estrategias y de la articulación y adaptación a los contextos y las realidades. Es por eso que para Aprende en Casa la familia, como primer sistema de socialización, cobra un especial protagonismo en diálogo con la escuela y el museo. El reto del programa ha sido, entre otros, intentar interpretar a familias tan diversas: rurales, urbanas, monoparentales, ampliadas, con diversos niveles socioeconómicos y de alfabetización.

La apuesta frente a dicho desafío va más allá de la familia como ayuda o como medio, sino como motor fundamental para la motivación del interés por el aprendizaje científico. Tomando a Melina Furman, 
el conocimiento se construye a partir del vínculo por el amor a aprender que, a su vez, se construya en los nińos y las niñas (2019). En este punto resulta primordial la idea del aprendizaje constante, no solo de niños y niñas, sino de la familia y, en este sentido, es importante en el diálogo con las familias motivar la conciencia sobre sus propios procesos de aprendizaje.

Por supuesto, eso lleva a muchas otras reflexiones como las expuestas durante este artículo: ¿qué rol debe tener la familia en esta presencialidad remota? Ya se ha identificado que se requiere una participación mucho más activa y con nuevas interacciones, pero sigue siendo necesario fortalecer, por ejemplo, la articulación familia-maestro y familia-museo más allá de la concertación de aspectos logísticos o informativos y del esparcimiento. También surge la duda sobre cómo brindar mayores oportunidades para que se dé la articulación curricular del quehacer cotidiano del profesor con los recursos, que tiene ahora un epicentro múltiple: escuela, casa y ciudad.

Esta reflexión acerca de la diversidad de las familias y de relaciones nos obliga a pensar en el tipo de material educativo que se debe diseñar y proponer para resolver esas necesidades en esos contextos y esas estructuras específicas. Pensar que las distancias solo se pueden acortar cuando media lo digital refuerza una idea particular que, a través de las brechas digitales, termina dejando por fuera del panorama a un gran número de familias cuya realidad no permite un acceso tan cómodo, fácil, efectivo o veloz a contenidos y materiales digitales o digitalizados. La responsabilidad que trae pensar en fortalecer el desarrollo de habilidades y actitudes científicas en este contexto de aislamiento implica hacerse esas preguntas y, eventualmente, terminar discutiendo con la "virtualización” de la educación como respuesta a la necesidad del aislamiento y el distanciamiento social.

El proceso pedagógico va más allá del ejercicio del diseño y la creación de materiales digitales. Implica también la reflexión del papel que juega cada actor (escuela, docentes, familia, museo, estudiantes) y cada tipo de recurso (digital o análogo) en estas estrategias de formación del pensamiento científico y la apropia- ción social del conocimiento científico y tecnológico. En términos generales, el ejercicio evaluativo con las instituciones focalizadas permitió identificar la alta valoración que se tiene del programa y el interés casi generalizado de participar nuevamente en él. En cuanto a los aspectos pedagógicos y de diseńo de los recursos educativos, hay una amplia satisfacción y es de resaltar la importancia de estos materiales para los niños y las posibilidades de articulación con el currículo. No obstante, frente a este último elemento se destaca que es necesario fortalecer los lazos con docentes y familias para un mayor aprovechamiento de los recursos educativos del programa. Se trata de una labor de doble vía, debido a que es necesario disponer de tiempos y espacios con los docentes para mejorar el proceso de articulación, así como desde Maloka proponer escenarios para que estas relaciones se estrechen.

Programas como este invitan también a pensar en los roles, desde su interacción con los materiales, de los estudiantes. Los procesos de desarrollo de pensamiento científico en niños y niñas experimentan transformaciones a través de la necesidad de implementar estrategias de presencialidad remota. Si la educación se reconoce como un proceso que ocurre en diversos lugares, surge inmediatamente el cuestionamiento de cómo se intenciona este proceso, con el nuevo ensamblaje de actores e intereses. El programa encuentra soluciones al pensar en las estrategias pedagógicas aquí presentadas y brinda opciones desde lo digital, pero también desde lo físico y lo virtual, así como del acompañamiento análogo (desde la línea educativa) hasta espacios digitales sincrónicos (franjas online) y asincrónicos (micrositio web). La transformación de la casa en una estación de exploración no solo trae estos retos anteriormente discutidos, sino que permite comenzar a entender las necesidades e, inclusive, empezar a resolverlas.

Esto es, de igual forma, revisar con mucho detenimiento si la pregunta a la hora de desarrollar materiales y recursos educativos para este panorama global es ¿̨cómo podemos virtualizar o digitalizar, de manera efectiva e incluyente, la educación?, o si debemos 
comenzar a cuestionarnos qué tipo de soluciones son realmente incluyentes y responden a una gama de necesidades tanto pedagógicas como de acceso. Es por esto que se insiste en que se discuta con mayor fuerza alrededor de estas materialidades que surgen como un espectro en donde la educación, frente al aislamiento y el confinamiento, exige mucho más que lo digital.

\section{Agradecimiento}

Agradecemos la lectura, los aportes y los comentarios de Marco Cardona Giraldo y Angélica Múnera Soto. Sus valiosas contribuciones ayudaron a madurar nuestros argumentos e ideas.

\section{Referencias}

Beetlestone, J. G., Johnson, C. H., Quin, M., \& White, H. (1998). The science center movement: context, practice, next challenges. Public Understand of Science, 1(7), 5-26. https://doi. org/10.1177/096366259800700101

Bradburne, J. M. (1998). Dinosaurs and White Elephants: the Science Centre in the 21st Century. Museum Management and Curatorship, 17(2), 119-137. https://doi.org/10.1080/ 09647779800201702

Coombs, P. (1971). La crisis mundial de la educación. Ediciones Península.

Daza-Caicedo, S., Maldonado, O., Arboleda-Castrillón, T., Falla, S., Moreno, P., Tafur-Sequera, M. \& Papagayo, D. (2017). Hacia la medición del impacto de las prácticas de apropiación social de la ciencia y la tecnología: Propuesta de una batería de indicadores. Historia, Ciencias, Saude - Manguinhos, 24(1), 145-164. https://doi.org/10.1590/S0104-5970201700 0100004

Dussel, I. (2020). La clase en pantuflas. En Inés Dussel, P. Ferrante \& D. Pulfer (Eds.), Pensar la educación en tiempos de pandemia : entre la emergencia, el compromiso y la espera (pp. 337-350). UNIPE: Editorial Universitaria.
Elizarrarás, O. (2020). Adaptarse o desaparecer. Comentario sobre las consecuencias de los museos por la pandemia de COVID-19, un panorama sin dinero, sin aglomeraciones y en línea. En Los museos y la pandemia (pp. 12-16), Universidad de Guanajuato.

Falk, J. \& Dierking, L. (2018). Viewing science learning through an ecosystem lens: A story in two parts. In Navigating the changing landscape of formal and informal science learning opportunities. C. Springer.

Franco-Avellaneda, M. (2013a). Ensamblar museus de ciências e tecnologias: compreensóes educativas a partir de três estudos de caso. [Tesis de Doctorado, UFSC, Florianópolis-Brasil].

Franco-Avellaneda, M. (2013b). Museos, artefactos y sociedad: ¡cómo se configura su dimensión educativa? Universitas Humanistica, 1(76), 125-151.

Franco-Avellaneda, M. \& Arboleda, T. (2014). Apropiación Social de la Ciencia, Tecnología e Innovación para el Desarrollo Humano. Escuela Virtual PNUD-Colciencias / Diplomado ASCTI.

Furman, M. (2016). Educar mentes curiosas: la formación del pensamiento cientifico y tecnológico en la infancia: documento básico, XI Foro Latinoamericano de Educación. En Santillana (Ed.), Espiral. Cuadernos Del Profesorado (1 a ed com, Vol. 10). https://doi.org/10.25115/ecp. v10i20.1015

Furman, M. (2019). Guia para criar hijos curiosos: Ideas para encender la chispa del aprendizaje. Siglo veintiuno editores.

García, S. (2020). COVID-19 y educación primaria y secundaria: repercusiones de la crisis e implicaciones de política pública para América Latina y el Caribe. PNUD, Serie de documentos de política pública. https://www.unicef.org/lac/informes/ covid-19-y-educacion-primaria-y-secundaria

Martínez-Boom, A. (2004). De la escuela expansiva a la escuela competitiva: Dos modos de modernización en América Latina. Anthropos-CAB. 
Ministerio de Tecnologías de la Información y las Comunicaciones. (2020). Uno de cada dos hogares no tiene internet móvil o fijo: ministra TIC sobre brecha digital. https://www. mintic.gov.co/portal/inicio/Sala-de-Prensa/ MinTIC-en-los-Medios/79575:Uno-de-cadados-hogares-no-tiene-internet-movil-o-fijoministra-TIC-sobre-brecha-digital

OECD. (2016). Reviews of National Policies for Education. Education in Colombia. OCDE.

Pérez-Bustos, T. (2009). Tan lejos... Tan cerca. Articulaciones entre la popularizaciòn de la ciencia y la tecnología y los sistemas educativos en Colombia. Interciencia, 34(11), 814-821.

Pérez-Bustos, T. (2010). Los márgenes de la popularización de la ciencia y la tecnología. Conexiones feministas en el sur global. [Tesis de Doctorado, Programa Interinstitucional, UPN-Univalle-UD, Bogotá D.C].
Porras, Y. (2021). La educación en ciencias en un escenario de pandemia. Tecné-Episteme-Didaxis, (49), 7-15.

Secretaría Distrital de Planeación. (2019). 097- Caracterización de vivienda y población de la zona rural de Bogotá D.C.

Tejedor, F. (2000). El diseño y los diseños en la evaluación de programas. Revista de Investigación Educativa, RIE, 18(2), 319-340.

Uniandes. (2020). COVID-19 acciones globales frente al cierre de escuelas durante una pandemia (J. García \& J. Kairuz, Eds.). Universidad de los Andes. 